\title{
RELATIONSHIP BETWEEN DCT-II, DCT-VI, AND DST-VII TRANSFORMS
}

\author{
Yuriy A. Reznik \\ InterDigital Communications \\ 9710 Scranton Rd, San Diego, CA 92121, USA \\ Email: yreznikeieee.org
}

\begin{abstract}
Discrete Sine Transforms of type VII (DST-VII) have recently received considerable interest in video coding. In this paper, we show that there exists a direct connection between DSTVII and DCT-II transforms, allowing their joint computation for certain transform sizes. This connection also yields fast algorithms for constructing DCT-VI and DCT-VII.
\end{abstract}

Index Terms- KLT, DCT-II, DCT-VI, DST-VII, factorizations, video coding.

\section{INTRODUCTION}

The Discrete Cosine Transforms of types II and IV are among most fundamental, well understood, and much appreciated tools in data compression. The DCT-II is used at the core of standards for image and video compression, such as JPEG, ITU-T H.26x-series, and MPEG 1-4 standards [1]. The DCT-IV is used in audio coding algorithms, such as ITU-T Rec. G.722.1, MPEG-4 AAC, and others [2]. Such transforms are very well studied, and a number of efficient technique exists for their computation [1, 3, 4, 5, 6, 7].

Much less known are so-called "odd" sinusoidal transforms: Discrete Cosine and Sine Transforms of types V, VI, VII, and VIII. Existence of some of such transforms was discovered by A. Jain in 1979 [8]. A complete tabulation was developed in 1985 by Wang and Hunt [9]. However, not much work has followed. Surveys of related results can be found in $[10,3]$.

Recently, DST of types VI and VII have surfaced as useful tools in image and video coding. In 2010, Han, Saxena, and Rose have shown that DST-VII produce good approximations of Karhunen-Loeve Transform (KLT) for model of residual signals after Intra-prediction [11]. This was subsequently validated in the course of experimental work on ISO/IEC/ITU-T High Efficiency Video Coding (HEVC) standard [12, 13].

The adoption of DST-VII in HEVC has prompted a discussion on the existence of fast algorithms for computing of such transforms $[12,14]$. This question was addressed in 2011 by Chivukula and Reznik[15], who have established connection between DST-VII and DFT.
This paper offers an alternative solution by establishing a mapping between DST-VII, DCT-VI, and DCT-II. This mapping yields fast algorithms not only for DST-VI/VII, but also for DCT-VI/VII, as well as possible joint factorizations of such transforms. The obtained mapping may also be of interest from methodological standpoint, as it suggests additional connections between DST-VII, DCT-VI and KLT of residual and mixed signals.

The rest of this paper is organized as follows. Section 2 provides definitions. Section 3 establishes mapping between DCT-II, DCT-VI and DST-VII transforms. Section 4 explains how this mapping can be used to construct fast algorithms. Discussion and concluding remarks are offered in Section 5.

\section{DEFINITIONS}

Let $N$ be the length of data sequence. The matrices of Discrete Fourier Transform (DFT) and Discrete Cosine and Sine transforms of types II, III, IV, VI, and VII will be defined as follows:

$\begin{array}{lll}\text { DFT: } & {\left[F_{N}\right]_{m n}=e^{-j \frac{2 \pi m n}{N}},} & m, n \in[0, N-1] \\ \text { DCT-II: } & {\left[C_{N}^{I I}\right]_{m n}=\cos \frac{m(2 n+1) \pi}{2 N},} & m, n \in[0, N-1] \\ \text { DCT-III: } & {\left[C_{N}^{I I I}\right]_{m n}=\cos \frac{(2 m+1) n \pi}{2 N},} & m, n \in[0, N-1] \\ \text { DCT-IV: } & {\left[C_{N}^{I V}\right]_{m n}=\cos \frac{\pi(2 m+1)(n+1)}{4 N},} & m, n \in[0, N-1] \\ \text { DCT-VI: } & {\left[C_{N+1}^{V I}\right]_{m n}=\cos \frac{m(2 n+1) \pi}{2 N+1},} & m, n \in[0, N] \\ \text { DCT-VII: }\left[C_{N+1}^{V I I}\right]_{m n}=\cos \frac{(2 m+1) n \pi}{2 N+1}, & m, n \in[0, N] \\ \text { DST-VI: } & {\left[S_{N}^{V I}\right]_{m n}=\sin \frac{(m+1)(2 n+1) \pi}{2 N+1},} & m, n \in[0, N-1] \\ \text { DST-VII: } & {\left[S_{N}^{V I I}\right]_{m n}=\sin \frac{(2 m+1)(n+1) \pi}{2 N+1},} & m, n \in[0, N-1]\end{array}$

In the above definitions, we have intentionally omitted normalization constants (such as $\sqrt{2 / N}$ and $\lambda_{i}=\left[\begin{array}{c}1 / \sqrt{2}, i=0 \text {, } \\ 1, i \neq 0\end{array}\right.$ conventionally used in definition of DCT-II) as they don't affect factorization structures of the transforms. Sub-indices $N$ or $N+1$ indicate lengths of the transforms. We follow Wang and Hunt's convention of coupling $N$-point DST-VI/VII with $N+1$-point DCT-VI/VII [9].

As easily noticed, transforms of types II and III, as well as VI and VII are closely related:

$$
\left(C_{N}^{I I}\right)^{T}=C_{N}^{I I I} ; \quad\left(C_{N+1}^{V I}\right)^{T}=C_{N+1}^{V I I} ; \quad\left(S_{N}^{V I}\right)^{T}=S_{N}^{V I I} .
$$




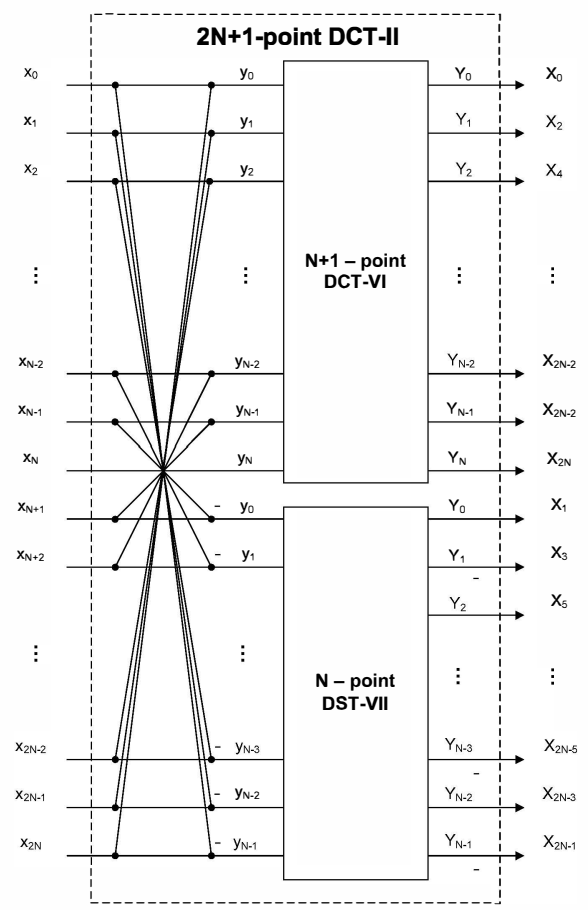

(a)

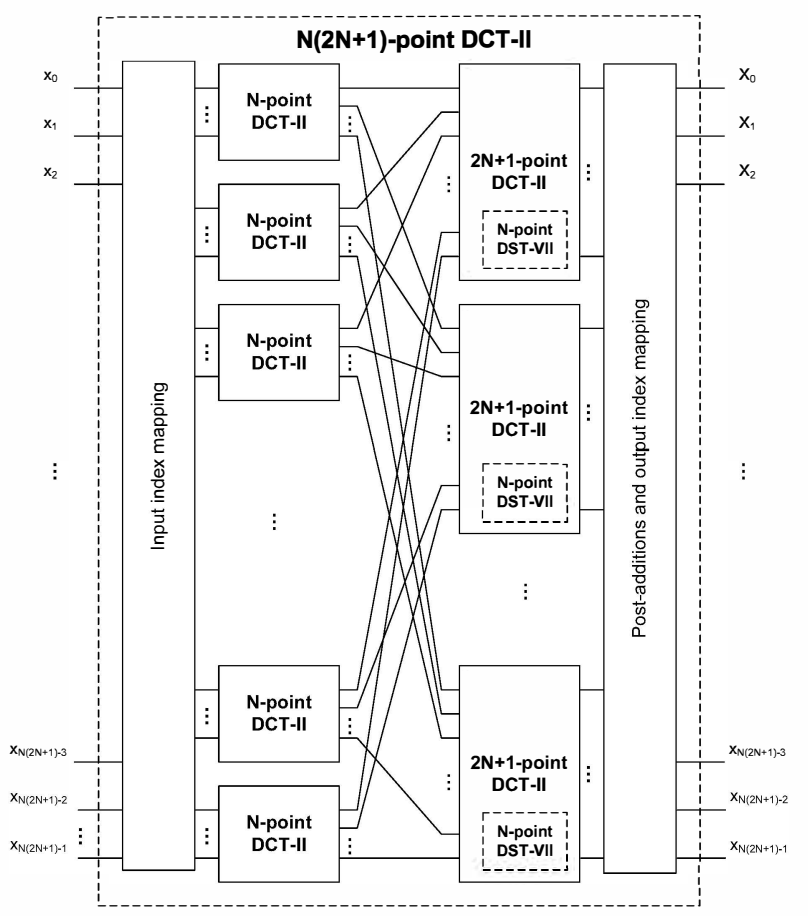

(b)

Fig. 1. Computing DCT-II of composite sizes: (a) split of $2 N+1$-point DCT-II into an $N+1$-point DCT-VI and $N$-point DST-VI; (b) computation of DCT-II of length $N(2 N+1)$.

\section{RELATIONSHIP BETWEEN $2 N+1$-POINT DCT-II, $N+1$-POINT DCT-VI, AND $N$-POINT DST-VII TRANSFORMS}

In this section we prove the following statement.

Theorem 1. The following holds:

$$
C_{2 N+1}^{I I}=Q_{2 N+1}\left(\begin{array}{cc}
C_{N+1}^{V I} & \\
& S_{N}^{V I I}
\end{array}\right)\left(\begin{array}{ccc}
I_{N} & & J_{N} \\
& 1 & \\
-J_{N} & & I_{N}
\end{array}\right)
$$

where $Q_{2 N+1}$ is a matrix, such that when applied to a vector $x$, it produces the following sign alterations and reordering:

$$
\begin{array}{lll}
\tilde{x}_{2 i} & =x_{i} & i=0, \ldots, N \\
\tilde{x}_{2 i+1}=(-1)^{i} x_{N+1+i} & i=0, \ldots, N-1,
\end{array}
$$

and $I_{N}$ and $J_{N}$ are $N \times N$ identity and order-reversal matrices respectively.

Proof. Let us consider a $2 N+1$-long input sequence $x=$ $x_{0}, \ldots, x_{2 N}$, and apply DCT-II over it:

$$
X_{k}^{C^{I I}}=\sum_{n=0}^{2 N} x_{n} \cos \frac{\pi(2 n+1) k}{2(2 N+1)}, k=0, \ldots, 2 N .
$$

We first look at even output values $(k=2 \mathrm{i}, \mathrm{i}=0, \ldots, \mathrm{N})$ :

$$
\begin{aligned}
X_{2 i}^{C^{I I}} & =\sum_{n=0}^{2 N} x_{n} \cos \frac{\pi(2 n+1) 2 i}{2(2 N+1)} \\
& =\sum_{n=0}^{2 N} x_{n} \cos \frac{\pi(2 n+1) i}{2 N+1}
\end{aligned}
$$

We split this sum as follows:

$$
\begin{aligned}
X_{2 i}^{C^{I I}} & -\sum_{n=0}^{N} x_{n} \cos \frac{\pi(2 n+1) i}{2 N+1} \\
& =\sum_{n=N+1}^{2 N} x_{n} \cos \frac{\pi(2 n+1) i}{2 N+1} \\
& =\sum_{n=N+1}^{2 N} x_{n} \cos \frac{\pi(2(2 N-n)+1) i}{2 N+1} \\
& =\sum_{n=0}^{N-1} x_{2 N-n} \cos \frac{\pi(2 n+1) i}{2 N+1},
\end{aligned}
$$

which implies that

$$
X_{2 i}^{C^{I I}}=X_{i}^{C^{V I}}+X_{i}^{\prime C^{V I}}
$$




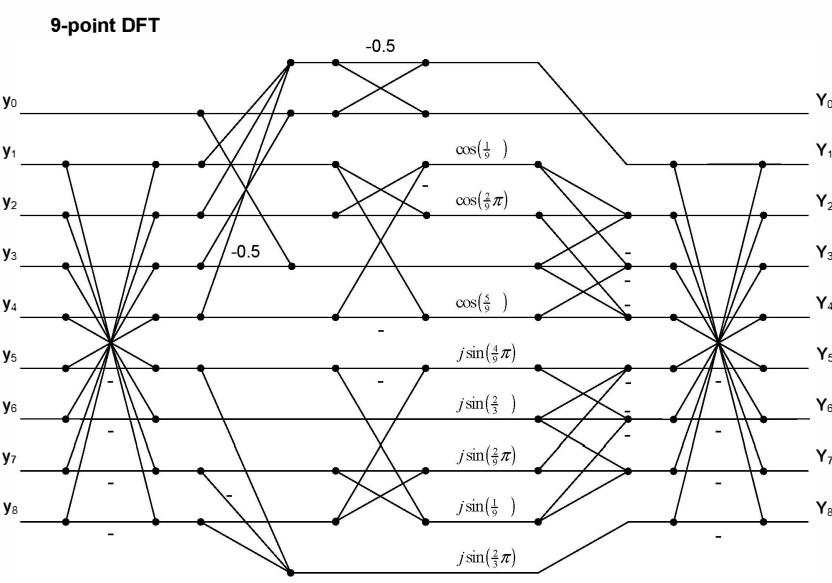

(a)

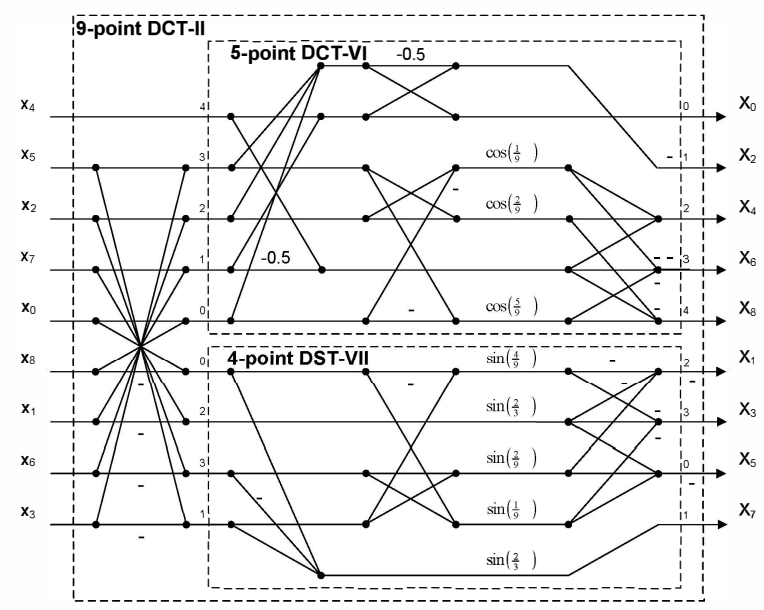

(b)

Fig. 2. Flow-graph of Winograd's factorization of DFT of length 9, and flow-graphs of 9-point DCT-II, 5-point DCT-VI, and 4-point DST-VII implied by mappings $(1,3)$.

where $X^{C^{V I}}$ is a DCT-VI transform over the first $N+1$ elements of input sequence $x$, and $X^{\prime C^{V I}}$ is a DCT-VI transform over the following input:

$$
x_{n}^{\prime}=\left[\begin{array}{ll}
x_{2 N-n}, & \text { if } n=0, \ldots, N-1, \\
0, & \text { if } n=N .
\end{array}\right.
$$

We now turn our attention to the odd output values $(k=$ $2 i+1, i=0, \ldots, N-1)$ :

$$
\begin{aligned}
X_{2 i+1}^{C^{I I}} & =\sum_{n=0}^{2 N} x_{n} \cos \frac{\pi(2 n+1)(2 i+1)}{2(2 N+1)} \\
& =(-1)^{i+1} \sum_{n=0}^{2 N} x_{2 N-n} \sin \frac{\pi(N-n)(2 i+1)}{2 N+1}
\end{aligned}
$$

We split this sum as follows:

$$
\begin{aligned}
& X_{2 i+1}^{C^{I I}}+(-1)^{i} \sum_{n=0}^{N-1} x_{2 N-n} \sin \frac{\pi(N-n)(2 i+1)}{2 N+1} \\
& =(-1)^{i+1} \sum_{n=N+1}^{2 N} x_{2 N-n} \sin \frac{\pi(N-n)(2 i+1)}{2 N+1} \\
& =(-1)^{i} \sum_{n=0}^{N-1} x_{N-1-n} \sin \frac{\pi(n+1)(2 i+1)}{2 N+1},
\end{aligned}
$$

which implies that

$$
X_{2 i+1}^{C^{I I}}=(-1)^{i+1} \tilde{X}_{i}^{S^{V I I}}+(-i)^{i} \hat{X}_{i}^{S^{V I I}}
$$

where $\tilde{X}^{S^{V I I}}$ is an $N$-point DST-VII transform over a sequence

$$
\tilde{x}_{n}=x_{N+1+n}, \quad n=0, \ldots, N-1,
$$

and $\hat{X}^{S^{V I I}}$ is an $N$-point DST-VII transform over a sequence

$$
\hat{x}_{n}=x_{N-1-n}, \quad n=0, \ldots, N-1 .
$$

By combining all these mappings we arrive at expression (1).

We present a flowgraph of the resulting mapping between DCT-II, DCT-VI, and DST-VII transforms in Figure 1.a. Only $2 N$ additions, permutations and sign changes are needed to convert output of DCT-VI, and DST-VII into DCT-II.

\section{FAST ALGORITHMS FOR COMPUTING DCT-II, DCT-VI, AND DST-VII}

\subsection{Connection to DFT}

From (1) it follows that fast computation of DCT-VI and DSTVII can be reduced to computing subsets of DCT-II. According to Heideman [4] it is also known that computing of DCTII of odd numbers is equivalent to computing same-length DFT. Considering $2 N+1$-point transforms, we can summarize Heideman's result as follows:

$$
C_{2 N+1}^{I I}=H_{1}\left(\begin{array}{l}
{\left[\Re\left(F_{2 N+1}\right)\right]_{\text {rows } 0, \ldots, N}} \\
{\left[\Im\left(F_{2 N+1}\right)\right]_{\text {rows } N+1, \ldots, 2 N}}
\end{array}\right) H_{2},
$$

where $\Re\left(F_{2 N+1}\right)$ and $\Im\left(F_{2 N+1}\right)$ denote real and imaginary parts of the DFT transform matrix of size $2 N+1$, and $H_{1}$ and $\mathrm{H}_{2}$ are some permutation and sign-inversion matrices [4].

In combination with (1) this formula shows that an $N+1$ point DCT-VI, an $N$-point DST-VII and an $2 N+1$-point DCT-II can be computed by mapping to a $2 N+1$-point DFT. Since many algorithms for computing of DFT are readily available (see e.g. [16]), this automatically leads to to fast algorithms for computing DCT-VI and DST-VII. 


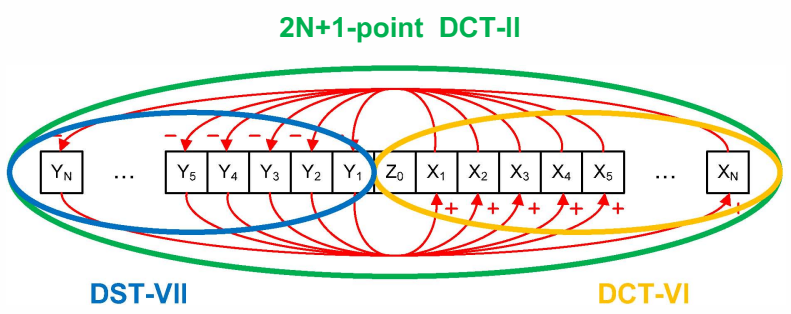

(a)

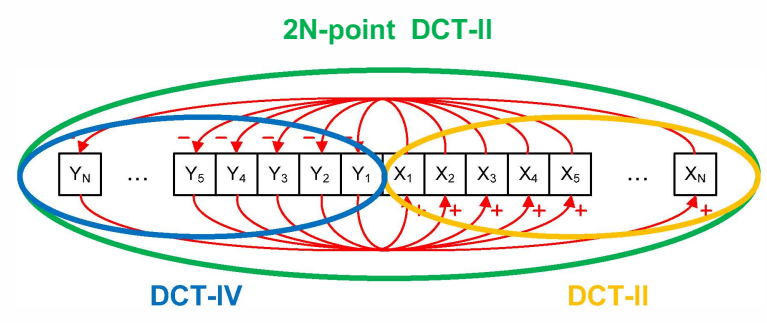

(b)

Fig. 3. Conceptual illustration of decompositions of (a) $2 N+1$-point DCT-II (1) and (b) $2 N$-point DCT-II (4).

\subsection{Examples of fast algorithms for $N=4$}

We use Winograd DFT module of length 9 shown in Figure 2.a. This particular factorization comes from [16]. By using this flowgraph and mappings (3) and (1) we easily obtain 9-point DCT-II, 5-point DCT-VI, and 4-point DST-VII. This is shown in Figure 2.b.

We note that all these algorithms are very efficient in terms of multiplicative complexity. Thus, obtained 9-point DCT-II requires only 8 non-trivial multiplications. In contrast, the least complex algorithms for computing DCT-II of size 8 (nearest dyadic-size) requires 11 multiplications[7].

The obtained 4-point DST-VII is also very efficient: it uses only 5 multiplications. This factorization is immediately suitable for implementing an integer approximation of DSTVII transform defined in HEVC standard [13].

Finally, factorization of a 5-point DCT-VI shown in Figure 2 .b needs only 3 real multiplications and 2 shifts (multiplications by factors $1 / 2$ ).

\subsection{Fast computing of transforms of length $2^{k} N(2 N+1)$}

It is known that a transform of a composite length $N=p q$, where $p$ and $q$ are co-prime, can be decomposed into a cascade of $p q$-point transforms and $q p$-point transforms followed by $p q-p-q-1$ additions. This class of techniques is called Prime Factor Algorithms (PFA) [17, 18].

In Figure 1.b, we show how to compute DCT-II of length $N(2 N+1)$. This factorization includes $2 N+1 N$-point DCTII sub-transforms, and additionally $N 2 N+1$-point DCTII transforms, which, in turn include $N$-point DST-VII as part of their flowgraph. Hence, a system that implements and uses $N$-point DCT-II and DST-VII, can easily compute an $N(2 N+1)$ transform by reusing them. Same principle more generally applies to computing transforms of lengths $2^{k} N(2 N+1)$.

Embedded factorization structures including DST-VII blocks in flowgraphs for DCT-II can be of interest to hardware implementations, as it offers potential for reducing the area, cost, and power usage of a circuit responsible for computing transforms.

\section{DISCUSSION AND CONCLUDING REMARKS}

We notice that decomposition (1) looks very similar to the well-known split of even-sized DCT-II (see, e.g. [3]):

$$
C_{2 N}^{I I}=P_{2 N}\left(\begin{array}{cc}
C_{N}^{I I} & \\
& C_{N}^{I V} J_{N}
\end{array}\right)\left(\begin{array}{cc}
I_{N} & J_{N} \\
J_{N} & -I_{N}
\end{array}\right),
$$

where $P_{2} N$ is a certain permutation matrix. This split leads to recursive construction due to reappearance of DCT-II in the upper part of decomposition. In contrast, our decomposition of $2 N+1$-point DCT-II (1) does not immediately lead to a recursion.

In Figure 3 we offer conceptual illustration of both decompositions (1) and (4). Input data samples are denoted as $y_{N}, \ldots, y_{1}, z_{0}, x_{1}, \ldots, x_{N}$ in a $2 N+1$-point case (a), and $y_{N}, \ldots, y_{1}, x_{1}, \ldots, x_{N}$ in $2 N$-point case (b). It is shown that the lower (right) portion of DCT-II transform becomes essentially equivalent to DST-VII (or DCT-IV) transform over residual samples $y_{i}^{\prime}=y_{i}-x_{i}$, while the upper (right) portion of DCT-II transform becomes essentially equivalent to DCT-VI (or DCT-II) transform over sums: $x_{i}^{\prime}=x_{i}+y_{i}$, $(i=1, \ldots, N)$. In the $2 N+1$-point case, the upper transform also absorbs the middle sample $z_{0}$.

This illustration may be insightful for understanding meanings of the involved transforms. For instance, in signal processing, it is customary to think of DCT-II as an approximation of KLT for 1-st order Markov source with high correlation coefficient. Decomposition in Figure 3.a shows that DST-VII, as well as DCT-VI (with some permutations and sign changes) can be understood as approximations of KLT over residual or mixed signals with progressively increasing distances between samples. Similarly, decomposition in Figure 3.b shows that in case of a $2 N$-sample arrangement, it is DCT-IV and DCT-II that can be understood as approximations of KLT over residual and mixed signals.

The obtained relationship (1) may also be instrumental in showing that DST-VII-based coding of Intra-prediction residual is essentially equivalent to performing L-shaped DCTII, where one part of L-shape corresponds to boundary pixels, and the other part absorbs pixels predicted based on this boundary. A design of direction-adaptive transforms based on similar idea was proposed in [19]. 


\section{REFERENCES}

[1] K. R. Rao and P. Yip, Discrete Cosine Transform: Algorithms, Advantages, Applications, Academic Press, Boston, MA, 1990.

[2] R. K. Chivukula and Y. A. Reznik, "Efficient implementation of a class of MDCT/IMDCT filterbanks for speech and audio coding applications," in IEEE Int. Conf. Acoust., Speech, Signal Processing (ICASSP), July 2008, pp. pp. 213-216.

[3] V. Britanak, K. R. Rao, and P. Yip, Discrete Cosine and Sine Transforms: General Properties, Fast Algorithms and Integer Approximations, Academic Press - Elsevier, Oxford, UK, 2007.

[4] M. T. Heideman, "Computation of an odd-length DCT from a real-valued DFT of the same length," IEEE Trans. Signal Processing, vol. 40, no. 1, pp. 54-61, 1992.

[5] C. W. Kok, "Fast algorithm for computing Discrete Cosine Transform," IEEE Trans. Signal Processing, vol. 45, no. 3, pp. 757-760, 1997.

[6] E. Feig and S. Winograd, "On the multiplicative complexity of Discrete Cosine Transforms (Corresp.)," IEEE Trans. Information Theory, vol. IT-38, pp. 1387-1391, 1992.

[7] C. Loeffler, A. Ligtenberg, and G.S. Moschytz, "Practical fast 1-D DCT algorithms with 11 multiplications," in IEEE Int. Conf. Acoust., Speech, Signal Processing (ICASSP), May 1989, vol. 2, pp. 988-991.

[8] A. K. Jain, "A sinusoidal family of unitary transforms," IEEE Trans. Pattern Analysis and Machine Intelligence, vol. PAMI1, no. 4, pp. $356-365$, Oct. 1979.

[9] Z. Wang and B. R. Hunt, "The discrete W transform," Applied Mathematics and Computation, vol. 16, no. 1, pp. 19 - 48, 1985.

[10] S. A. Martucci, "Symmetric convolution and the Discrete Sine and Cosine Transforms," IEEE Trans. Signal Processing, vol. SP-42, pp. 1038-1051, 1994.

[11] J. Han, A. Saxena, and K. Rose, "Towards jointly optimal spatial prediction and adaptive transform in video/image coding," in IEEE Int. Conf. Acoust., Speech, Signal Processing (ICASSP), March 2010, pp. 726-729.

[12] A. Saxena and F. Fernandes, "CE7: Mode-dependent DCT/DST for intra prediction in video coding," commitee input document JCTVC-D033, ISO/IEC/ITU-T Joint Collaborative Team on Video Coding, Daegu, Korea, January 2011.

[13] B. Bross, W-J. Han, G. Sullivan, J. Ohm, and T. Wiegland, "High Efficiency Video Coding (HEVC) text specification draft 6," ITU-T and ISO/IEC JCTVC-HIOO3, Feb 2012.

[14] F. Fernandes A. Saxena and Y. Reznik, "Fast transforms for intra-prediction-based image and video coding," in Data Compression Conference (DCC), March 2013 - submitted.

[15] R. K. Chivukula and Y. A. Reznik, "Fast computing of discrete cosine and sine transforms of types vi and vii," in Applications of Digital Image Processing XXXIV, A. G. Tescher, Ed., 2011, vol. 8135 of Proc. SPIE, pp. 813505-1-14.
[16] C. S. Burrus and T. W. Parks, DFT/FFT and Convolution Algorithms and Implementation, Wiley-Interscience, New York, 1985.

[17] P.P.N. Yang and M.J. Narasimha, "Prime factor decomposition of the discrete cosine transform," in IEEE Int. Conf. Acoust., Speech, Signal Processing (ICASSP), March 1985, pp. pp. $772-775$.

[18] B. G. Lee, "Input and output index mapping for a prime-factor decomposed computation of discrete cosine transform," IEEE Trans. Acoust., Speech, Signal Processing, vol. 37, no. 2, pp. 237 - 244, Feb. 1989.

[19] S. S. Tsai C-L. Chang, M. Makar and B. Girod, "Directionadaptive partitioned block transform for color image coding," IEEE Transactions on Image Processing, , no. 7, pp. 17401755, July 2010. 